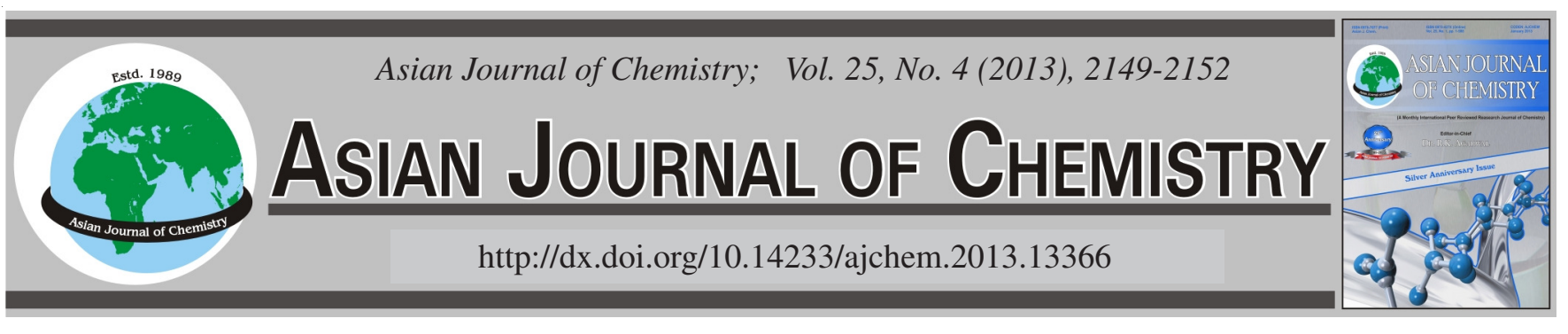

\title{
Synthesis and Herbicidal Activity of Amide Derivatives Containing Thiazole Moiety
}

\author{
JiAn-QuAn Weng ${ }^{1, *}$, XING-Hai LiU ${ }^{1}$ and GuO-Tong Tong ${ }^{2}$
}

${ }^{1}$ College of Chemical Engineering and Materials Science, Zhejiang University of Technology, Hangzhou 310014, Zhejiang Province, P.R. China ${ }^{2}$ Department of Chemical Engineering, Hangzhou Vocational and Technical College, Hangzhou 310018, Zhejiang Province, P.R. China

*Corresponding author: Tel: +86 571 88320220; E-mail: jqweng@zjut.edu.cn

(Received: 30 December 2011;

Accepted: 17 October 2012)

AJC-12300

Twelve novel amide derivatives containing thiazole moiety were synthesized via a coupling reaction of [4-(substituted phenyl)thiazol-2-
yl] acetonitrile and aryl isocyanates catalyzed by organic bases. Their structures were characterized by ${ }^{1} \mathrm{H}$ NMR, FTIR, MS and elemental
analysis. The preliminary bioassay indicated that these compounds exhibited moderate herbicidal activities against Echinochloa crusgalli
and Amaranthus ascedense.
Key Words: Synthesis, Amide derivative, Thiazole, Herbicidal activity.

\section{INTRODUCTION}

Nitrogen and sulfur linked heterocyclic compounds have recently attracted considerable attention owing to their prominent biological activity ${ }^{1-4}$. As one of the potent heterocyclic compounds, thiazole derivatives played an important role in the field of novel agrochemicals because of their wide biological activity, such as insecticidal ${ }^{5}$, fungicidal ${ }^{6}$, antiviral ${ }^{7}$, herbicidal $^{8}$ and plant-growth-regulating ${ }^{9}$ activities. Meanwhile, it is reported that amides possess a diverse range of bioactivities in agrochemical field, for example insecticidal ${ }^{10}$, ungicidal $^{11}$, herbicidal $^{12}$ and acaricidal ${ }^{13}$ activity.

It is reported that the isocyanates displayed high activities. Carbamate derivatives can be easily prepared from isocyanates readily react with an proton in certain compounds, such as hydrogen of acids, alcohols and amines ${ }^{14}$. However, the reaction of isocyanates with hydrogen of methylene group have seldom been reported.

In view of these facts and also as a part of our work on the development of bioactive heterocyclic compounds, twelve novel amide derivatives containing thiazole moiety (5a-l) were synthesized via a coupling reaction of [4-(substituted phenyl) thiazol-2-yl] acetonitrile and aryl isocyanates catalyzed by organic bases. Their structures were characterized by ${ }^{1} \mathrm{H}$ NMR, FTIR, MS and elemental analysis and their herbicidal activities were also evaluated.

\section{EXPERIMENTAL}

All the solvents and other chemicals were used as received from different commercial sources without further purification.
2-Bromo-1-(4-fluorophenyl)ethanone $(\mathbf{1 a})^{15}$, [4-(4-fluorophenyl)thiazol-2-yl]acetonitrile (3a) $)^{15}$, 2-bromo-1-(2,6-difluorophenyl)ethanone $(\mathbf{1 b})^{16}, 2$-cyanothioacetamide $(\mathbf{2})^{16}$ and [4(2,6-difluorophenyl)thiazol-2-yl] acetonitrile $(\mathbf{3 b})^{16}$ were prepared according to literature procedures. Melting points were conducted on a X-4 melting-point apparatus and are uncorrected. The ${ }^{1} \mathrm{H}$ NMR spectra were recorded on Bruker ADVANCE III instrument (500MHz) using TMS as an internal standard and DMSO- $d_{6}$ as solvents. FTIR spectra were recorded on a NICOLET 6700 instrument. Mass spectra (ESI-MS) were recorded on a Therm LCQ TM Deca XP plus instrument. Elemental analyses were performed on a Vario EL elemental analyzer.

General procedure for the synthesis of aryl isocyanates (4): To a solution of bis(trichloromethyl)carbonate (1.5 g, 5 mmol) in toluene $(20 \mathrm{~mL})$ with constant stirring at $0{ }^{\circ} \mathrm{C}$ was added dropwise substituted aniline $(15 \mathrm{mmol})$ in toluene (10 $\mathrm{mL})$. The reaction mixture was continued to be stirred at 0-5 ${ }^{\circ} \mathrm{C}$ for $1 \mathrm{~h}$ and then heated to reflux until the solution turned clear. The solution was then refluxed for another $1 \mathrm{~h}$. After cooling, the mixture was then evaporated in vacuo to obtain corresponding aryl isocyanates (4) without further purification.

General procedure for the synthesis of the title amide derivatives (5a-1): [4-(Substituted phenyl)thiazol-2-yl] acetonitrile (10 mmol) and 4-dimethylamiopyridine (DMAP) (1 mmol) was dissolved in 1,4-dioxane $(40 \mathrm{~mL})$ and aryl isocyanate (12 mmol) was added dropwise to the mixture, then the final mixture was refluxed for $12 \mathrm{~h}$. The precipitate formed was filtered and recrystallized from ethanol to give the amide derivatives containing thiazole moeity (5a-1). 
N-Phenyl-2-cyano-2-(4-(4-fluorophenyl)thiazol-2yl)acetamide (5a): White solid, yield 82.2 \%. m.p. 189-191 ${ }^{\circ} \mathrm{C} ;{ }^{1} \mathrm{H}$ NMR (DMSO- $d_{6}$ ) $\delta: 4.04$ (s, 1H, NH), 7.01-7.54 (m, 9H, ArH), 7.17 (s, 1H, SCH), 11.57 (s, 1H, CHCN); IR (KBr, $\left.v_{\max }, \mathrm{cm}^{-1}\right): 3318(\mathrm{~N}-\mathrm{H}), 2179(\mathrm{CN}), 1712(\mathrm{C}=\mathrm{O}), 1639,1453$ (Ar); MS (ESI) m/z: $338\left([\mathrm{M}+\mathrm{H}]^{+}\right)$. Elemental analysis (\%), calcd. for $\mathrm{C}_{18} \mathrm{H}_{12} \mathrm{~N}_{3} \mathrm{OSF}$ : C, 64.08; H, 3.59; N, 12.46; found: C, 64.17; H, 3.47; N, 12.54 .

N-m-Tolyl-2-cyano-2-(4-(4-fluorophenyl)thiazol-2yl)acetamide (5b): White solid, yield 76.5 \%, m.p. 213-215 ${ }^{\circ} \mathrm{C}$; ${ }^{1} \mathrm{H}$ NMR (DMSO-d $)$ d: 2.33 (s, 3H, $\mathrm{CH}_{3}$ ), 4.02 (s, 1H, $\mathrm{NH}), 7.11-7.36$ (m, 8H, ArH), 7.37 (s, 1H, SCH), 11.63 (s, $1 \mathrm{H}, \mathrm{CHCN})$; IR $\left(\mathrm{KBr}, \mathrm{v}_{\max }, \mathrm{cm}^{-1}\right): 3302(\mathrm{~N}-\mathrm{H}), 2176(\mathrm{CN})$, $1708(\mathrm{C}=\mathrm{O}), 1637,1450(\mathrm{Ar})$; MS (ESI) m/z: $352\left([\mathrm{M}+\mathrm{H}]^{+}\right)$; Elemental analysis (\%), calcd. for $\mathrm{C}_{19} \mathrm{H}_{14} \mathrm{~N}_{3} \mathrm{OSF}$ : C, 64.94; $\mathrm{H}$, 4.02; N, 11.96; found: C, 64.87; H, 3.92; N, 12.05 .

N-p-Tolyl-2-cyano-2-(4-(4-fluorophenyl)thiazol-2yl)acetamide (5c): White solid, yield 79.0 \%, m.p. 193-195 ${ }^{\circ} \mathrm{C} ;{ }^{1} \mathrm{H}$ NMR (DMSO-d $)$ ) $2.31\left(\mathrm{~s}, 3 \mathrm{H}, \mathrm{CH}_{3}\right), 4.03$ (s, $1 \mathrm{H}$, NH), 7.09-7.32 (m, 8H, ArH), 7.33 (s, 1H, SCH), 11.59 (s, $1 \mathrm{H}, \mathrm{CHCN})$; IR $\left(\mathrm{KBr}, \mathrm{v}_{\max }, \mathrm{cm}^{-1}\right): 3307(\mathrm{~N}-\mathrm{H}), 2172(\mathrm{CN})$, $1702(\mathrm{C}=\mathrm{O}), 1637,1452(\mathrm{Ar}) ; \mathrm{MS}(\mathrm{ESI}) \mathrm{m} / \mathrm{z}: 352\left([\mathrm{M}+\mathrm{H}]^{+}\right)$; Elemental analysis (\%), calcd. for $\mathrm{C}_{19} \mathrm{H}_{14} \mathrm{~N}_{3} \mathrm{OSF}$ : C, 64.94; $\mathrm{H}$, 4.02; N, 11.96; found: C, 65.03; H, 3.87; N, 12.10 .

N-(2-Chlorophenyl)-2-cyano-2-(4-(4-fluorophenyl)thiazol-2-yl)acetamide (5d): White solid, yield $83.0 \%$, m.p. 189-191 ${ }^{\circ} \mathrm{C} ;{ }^{1} \mathrm{H}$ NMR (DMSO- $d_{6}$ ) $\delta: 4.00$ (s, $1 \mathrm{H}, \mathrm{NH}$ ), 7.047.52 (m, 8H, ArH), 7.34 (s, 1H, SCH), 11.70 (s, 1H, CHCN); IR $\left(\mathrm{KBr}, v_{\max }, \mathrm{cm}^{-1}\right): 3305(\mathrm{~N}-\mathrm{H}), 2183(\mathrm{CN}), 1704(\mathrm{C}=\mathrm{O})$, 1640, $1452(\mathrm{Ar}) ; \mathrm{MS}(\mathrm{ESI}) \mathrm{m} / \mathrm{z}: 372\left([\mathrm{M}+\mathrm{H}]^{+}\right)$; elemental analysis (\%), calcd. for $\mathrm{C}_{18} \mathrm{H}_{11} \mathrm{~N}_{3} \mathrm{OSClF}$ : C, 58.15; H, 2.98; N, 11.30; found: C, 58.27; H, 3.08; N, 11.47.

N-(3-Chlorophenyl)-2-cyano-2-(4-(4-fluorophenyl)thiazol-2-yl)acetamide (5e): White solid, yield $80.7 \%$, m.p. 193-195 ${ }^{\circ} \mathrm{C}$; ${ }^{1} \mathrm{H}$ NMR (DMSO- $d_{6}$ ) $\delta: 4.01$ (s, 1H, NH), 7.127.63 (m, 8H, ArH), 7.37 (s, 1H, SCH), 11.62 (s, 1H, CHCN); IR $\left(\mathrm{KBr}, \mathrm{v}_{\max }, \mathrm{cm}^{-1}\right): 3312(\mathrm{~N}-\mathrm{H}), 2162(\mathrm{CN}), 1710(\mathrm{C}=\mathrm{O})$, 1640, $1450(\mathrm{Ar}) ; \mathrm{MS}(\mathrm{ESI}) \mathrm{m} / \mathrm{z}: 372\left([\mathrm{M}+\mathrm{H}]^{+}\right)$; elemental analysis (\%), calcd. for $\mathrm{C}_{18} \mathrm{H}_{11} \mathrm{~N}_{3} \mathrm{OSClF}$ : C, 58.15; H, 2.98; N, 11.30; found: C, 58.22; H, 2.83; N, 11.50.

N-(4-Chlorophenyl)-2-cyano-2-(4-(4-fluorophenyl)thiazol-2-yl)acetamide (5f): White solid, yield $83.5 \%$, m.p. 198-200 ${ }^{\circ} \mathrm{C}$; ${ }^{1} \mathrm{H}$ NMR (DMSO- $d_{6}$ ) $\delta: 4.00$ (s, $1 \mathrm{H}, \mathrm{NH}$ ), 7.047.50 (m, 8H, ArH), 7.36 (s, 1H, SCH), 11.55 (s, 1H, CHCN); IR $\left(\mathrm{KBr}, v_{\max }, \mathrm{cm}^{-1}\right): 3310(\mathrm{~N}-\mathrm{H}), 2179(\mathrm{CN}), 1715(\mathrm{C}=\mathrm{O})$, 1638, $1450(\mathrm{Ar}) ; \mathrm{MS}(\mathrm{ESI}) \mathrm{m} / \mathrm{z}: 372\left([\mathrm{M}+\mathrm{H}]^{+}\right)$; elemental analysis (\%), calcd. for $\mathrm{C}_{18} \mathrm{H}_{11} \mathrm{~N}_{3} \mathrm{OSClF}$ : C, 58.15; $\mathrm{H}, 2.98 ; \mathrm{N}$, 11.30; found: C, 58.31; H, 3.13; N, 11.43.

N-(3-(Trifluoromethyl)phenyl)-2-cyano-2-(4-(4-fluorophenyl)thiazol-2-yl)acetamide (5g): White solid, yield 85.2 $\%$, m.p. $189-192{ }^{\circ} \mathrm{C} ;{ }^{1} \mathrm{H}$ NMR (DMSO- $\left.d_{6}\right) \delta: 4.02$ (s, $1 \mathrm{H}, \mathrm{NH}$ ), 7.13-7.65 (m, 8H, ArH), 7.40 (s, 1H, SCH), 11.63 (s, 1H, CHCN); IR (KBr, $\left.v_{\max }, \mathrm{cm}^{-1}\right): 3306(\mathrm{~N}-\mathrm{H}), 2163(\mathrm{CN}), 1708$ $(\mathrm{C}=\mathrm{O}), 1632,1450(\mathrm{Ar})$; MS (ESI) m/z: $406\left([\mathrm{M}+\mathrm{H}]^{+}\right)$; elemental analysis (\%), calcd. for $\mathrm{C}_{19} \mathrm{H}_{11} \mathrm{~N}_{3} \mathrm{OSF}_{4}$ : C, 56.30; $\mathrm{H}$, 2.74; N, 10.37; found: C, 56.51; H, 2.66; N, 10.53.

N-(4-(Trifluoromethyl)phenyl)-2-cyano-2-(4-(4-fluorophenyl)thiazol-2-yl)acetamide (5h): White solid, yield 81.6 $\%$, m.p. $187-189^{\circ} \mathrm{C} ;{ }^{1} \mathrm{H}$ NMR (DMSO- $\left.d_{6}\right) \delta: 4.00$ (s, $1 \mathrm{H}, \mathrm{NH}$ ),
7.15-7.63 (m, 8H, ArH), 7.39 (s, 1H, SCH), 11.62 (s, 1H, CHCN); IR (KBr, $\left.v_{\max }, \mathrm{cm}^{-1}\right): 3305(\mathrm{~N}-\mathrm{H}), 2165(\mathrm{CN}), 1712$ $(\mathrm{C}=\mathrm{O}), 1634,1452$ (Ar); MS (ESI) m/z: $406\left([\mathrm{M}+\mathrm{H}]^{+}\right)$; elemental analysis (\%), calcd. for $\mathrm{C}_{19} \mathrm{H}_{11} \mathrm{~N}_{3} \mathrm{OSF}_{4}$ : C, 56.30; $\mathrm{H}$, 2.74; N, 10.37; found: C, 56.47; H, 2.79; N, 10.50.

N-Phenyl-2-cyano-2-(4-(2,6-difluorophenyl)thiazol-2yl)acetamide (5i): White solid, yield $83.9 \%$, m.p. $193-195{ }^{\circ} \mathrm{C}$; ${ }^{1} \mathrm{H}$ NMR (DMSO-d $)$ ) 4.03 (s, 1H, NH), 7.05-7.54 (m, 8H, $\mathrm{ArH}), 7.15$ (s, 1H, SCH), 11.57 (s, 1H, CHCN); IR (KBr, $v_{\max }$, $\left.\mathrm{cm}^{-1}\right)$ : $3308(\mathrm{~N}-\mathrm{H}), 2176(\mathrm{CN}), 1711(\mathrm{C}=\mathrm{O}), 1646,1452(\mathrm{Ar})$; MS (ESI) m/z: $356\left([\mathrm{M}+\mathrm{H}]^{+}\right)$; elemental analysis (\%), calcd. for $\mathrm{C}_{18} \mathrm{H}_{11} \mathrm{~N}_{3} \mathrm{OSF}_{2}$ : C, 60.84; H, 3.12; N, 11.82; found: C, 60.86; $\mathrm{H}, 3.10 ; \mathrm{N}, 11.84$.

N-o-Tolyl-2-cyano-2-(4-(2,6-difluorophenyl)thiazol-2yl)acetamide (5j): White solid, yield $78.6 \%$, m.p. $203-205^{\circ} \mathrm{C}$; ${ }^{1} \mathrm{H}$ NMR (DMSO- $\left.d_{6}\right) \delta: 2.35$ (s, 3H, $\left.\mathrm{CH}_{3}\right), 4.02$ (s, 1H, NH), 6.97-7.40 (m, 7H, ArH), 7.35 (s, 1H, SCH), 11.52 (s, 1H, CHCN); IR (KBr, $\left.v_{\max }, \mathrm{cm}^{-1}\right): 3306(\mathrm{~N}-\mathrm{H}), 2173(\mathrm{CN}), 1715$ $(\mathrm{C}=\mathrm{O}), 1640,1450(\mathrm{Ar})$; MS (ESI) $\mathrm{m} / \mathrm{z}: 340.0\left([\mathrm{M}+\mathrm{H}]^{+}\right)$; elemental analysis (\%), calcd. for $\mathrm{C}_{19} \mathrm{H}_{13} \mathrm{~N}_{3} \mathrm{OSF}_{2}$ : C, 61.78; $\mathrm{H}$, 3.55; N, 11.38; found: C, 61.89; H, 3.42; N, 11.54 .

N-(4-Chlorophenyl)-2-cyano-2-(4-(2,6-difluorophenyl)thiazol-2-yl)acetamide (5k): White solid, yield $84.0 \%$, m.p. 198-200 ${ }^{\circ} \mathrm{C}$; ${ }^{1} \mathrm{H}$ NMR (DMSO- $\left.d_{6}\right) \delta: 4.00$ (s, 1H, NH), 7.087.52 (m, 7H, ArH), 7.38 (s, 1H, SCH), 11.59 (s, 1H, CHCN); IR $\left(\mathrm{KBr}, \mathrm{v}_{\max }, \mathrm{cm}^{-1}\right)$ : $3308(\mathrm{~N}-\mathrm{H}), 2179(\mathrm{CN}), 1709(\mathrm{C}=\mathrm{O})$, 1636, $1450(\mathrm{Ar}) ; \mathrm{MS}$ (ESI) m/z: $390\left([\mathrm{M}+\mathrm{H}]^{+}\right)$; elemental analysis (\%), calcd. for $\mathrm{C}_{18} \mathrm{H}_{10} \mathrm{~N}_{3} \mathrm{OSClF}_{2}$ : C, 55.46; H, 2.59; N, 10.78; found: C, 55.79; H, 2.56; N, 10.54.

N-(4-(Trifluoromethyl)phenyl)-2-cyano-2-(4-(2,6difluorophenyl)thiazol-2-yl)acetamide (5l): White solid, yield $85.8 \%$, m.p. $194-196{ }^{\circ} \mathrm{C} ;{ }^{1} \mathrm{H}$ NMR (DMSO- $d_{6}$ ) $\delta: 4.01$ (s, 1H, NH), 7.13-7.62 (m, 7H, ArH), 7.41 (s, 1H, SCH), 11.66 (s, 1H, CHCN); IR (KBr, $\left.v_{\max }, \mathrm{cm}^{-1}\right)$ : $3312(\mathrm{~N}-\mathrm{H}), 2181(\mathrm{CN})$, $1720(\mathrm{C}=\mathrm{O}), 1640,1452(\mathrm{Ar}) ; \mathrm{MS}(\mathrm{ESI}) \mathrm{m} / \mathrm{z}: 424\left([\mathrm{M}+\mathrm{H}]^{+}\right)$; elemental analysis (\%), calcd. for $\mathrm{C}_{19} \mathrm{H}_{10} \mathrm{~N}_{3} \mathrm{OSF}_{5}$ : C, 53.90; $\mathrm{H}$, 2.39; N, 9.93; found: C, 54.07; H, 2.36; N, 10.11 .

Bioassay of herbicidal activities: The solutions of the title compounds 5 (100 mg/L) were prepared by dissolving them in N,N-dimethylformamide with the addition of a little Tween- 80 and proper water. There were three replicates for each treatment. The mixture of the same amount of N,Ndimethylformamide, Tween- 80 and water was used as the control (CK). Herbicidal testing of the title compounds $\mathbf{5}$ was carried out in a plant growth room. Temperature $(24 \pm 1){ }^{\circ} \mathrm{C}$, RH $60 \pm 5 \%$, light intensity $10 \mathrm{Klux}$, photoperiod $8 \mathrm{~h} /$ day. Twenty seeds of each one of weed species including Echinochloa crusgalli and Amaranthus ascedense were chosen for testing. Seedlings were grown in the test plate of $9 \mathrm{~cm}$ diameter containing two pieces of filter paper and $9 \mathrm{~mL}$ solution of the tested compound $(100 \mathrm{mg} / \mathrm{L})$. The herbicidal activity was assessed as the inhibition rate in comparison with the control. The herbicidal rating score based on visual observation. Range from 0$100 \%, 0 \%$ means no effect, $100 \%$ means complete killing.

\section{RESULTS AND DISCUSSION}

Twelve novel amide derivatives containing thiazole moiety (5) were synthesized from a coupling reaction of 
[4-(substituted phenyl)thiazol-2-yl]acetonitrile (3) and aryl isocyanates (4) catalyzed by organic bases according to the route as shown in Scheme-I and the yields were not optimized.

It is found that [4-(4-fluorophenyl)thiazol-2-yl]acetonitrile (3a) can react with phenyl isocyanate in presence of the 4-dimethylamiopyridine (DMAP) to give the condensed product $\mathbf{5 a}$ in high yield in the described reaction conditions (Scheme-II, Table-1, entry 4). The use of other organic bases, such as 1,8-diazabicyclo[5,4,0]undec-7-ene (DBU), triethylamine $\left(\mathrm{Et}_{3} \mathrm{~N}\right)$, diethylamine $\left(\mathrm{Et}_{2} \mathrm{NH}\right)$, pyridine $(\mathrm{Py})$ and the condensing reagent 1,3-dicyclohexylcarbodiimide (DCC) gave only low or moderate yields of $\mathbf{5 a}$ (Table-1, entry 1-3,5,6). No reactions occurred without the base catalysts. Thus, the reactions of $\mathbf{3}$ with other aryl isocyanates were occurred at refluxing temperature in the presence of DMAP, leading to the desired compounds (5b-5l) in 76.5-85.8 \% yields. It is notably that these compouds were synthesized from a coupling reaction of aryl isocyanates with hydrogen of methylene $\left(\mathrm{CH}_{2}\right)$, which have seldom been reported. All the compounds were identified and characterized by ${ }^{1} \mathrm{H}$ NMR, FTIR, MS and elemental analysis.

\begin{tabular}{ccc}
\multicolumn{4}{c}{ TABLE-1 } \\
\multicolumn{3}{|c}{ ORGANIC BASES CATALYZED REACTIONS } \\
OF COMPOUND 3a WITH PhNCO \\
\hline Entry & Organic base & Isolated yield (\%) \\
\hline 1 & DBU & 5.6 \\
2 & $\mathrm{Et}_{3} \mathrm{~N}$ & 64.5 \\
3 & $\mathrm{Et}_{2} \mathrm{NH}$ & 43.8 \\
4 & $\mathrm{DMAP}$ & 82.2 \\
5 & $\mathrm{Py}$ & 69.0 \\
6 & $\mathrm{DCC}$ & 32.2 \\
\hline
\end{tabular}

In the infrared spectrum (FT-IR) of compound 5, N-H stretching absorption signal appears at $3318-3302 \mathrm{~cm}^{-1}$ and the characteristic stretching vibration signals of $\mathrm{v}(\mathrm{C}=\mathrm{O})$ appears at 1720-1702 $\mathrm{cm}^{-1}$. In the ${ }^{1} \mathrm{H}$ NMR spectrum of $\mathbf{5}$, the -NH proton signals were observed at about $4.00 \mathrm{ppm}$ as a singlet and the $-\mathrm{CH}$ (linked cyano) proton signals were observed at
11.52-11.70 ppm as a singlet due to the influence of both electron-withdrawing groups cyano $(-\mathrm{CN})$ and carbonyl $(-\mathrm{C}=\mathrm{O})$, which indicated the conversion of $-\mathrm{C}=\mathrm{C}-\mathrm{OH}$ group.

Herbicidal activities: The herbicidal activity of all compounds $\mathbf{5}$ against Echinochloa crus-galli (barnyard grass) and Amaranthus ascedense (amaranthus lividus) has been investigated at the dosage of $100 \mathrm{mg} / \mathrm{L}$ using known procedure ${ }^{17}$ compared with the control $(\mathrm{CK})$. The results of herbicidal activity testing are listed in Table-2. The results of bioassay showed that all compounds exhibited moderate herbicidal activity against Echinochloa crusgalli and Amaranthus ascedense at the concentration of $100 \mathrm{mg} / \mathrm{L}$ and some of them exhibited moderate herbicidal activity against the root of Echinochloa crus-galli. For example, compounds 5d, 5f, 5g, $\mathbf{5 h}$ and $\mathbf{5 l}$ showed $>70 \%$ inhibitory rate to root of Echinochloa crus-galli. It is worthy to note that the compounds bearing an electron-withdrawing group at the benzene ring (e.g., $\mathbf{5 d}, \mathbf{5 e}$, $\mathbf{5 f}, \mathbf{5 g}, \mathbf{5 h}$ ) displayed higher herbicidal activity against the root of Echinochloa crusgalli than those compounds bearing an electron-donating group (e.g., 5b, 5c).

\begin{tabular}{|c|c|c|c|c|}
\hline \multicolumn{5}{|c|}{$\begin{array}{c}\text { TABLE-2 } \\
\text { HERBICIDAL ACTIVITIES OF TITLE } \\
\text { COMPOUNDS 5a-l AT } 100 \text { mg/L }\end{array}$} \\
\hline \multirow{3}{*}{ Compounds } & \multicolumn{4}{|c|}{ Relative inhibition $(\%)$} \\
\hline & \multicolumn{2}{|c|}{ Echinochloa crusgalli } & \multicolumn{2}{|c|}{ Amaranthus ascedense } \\
\hline & Root & Stalk & Root & Stalk \\
\hline $5 a$ & 57.8 & 43.6 & 55.3 & 45.0 \\
\hline $5 b$ & 54.4 & 51.8 & 52.6 & 41.2 \\
\hline $5 c$ & 52.4 & 43.6 & 49.5 & 29.5 \\
\hline $5 d$ & 73.2 & 47.3 & 58.2 & 33.3 \\
\hline $5 e$ & 69.8 & 39.5 & 54.9 & 32.0 \\
\hline $5 f$ & 73.5 & 50.0 & 56.7 & 37.5 \\
\hline $5 g$ & 78.0 & 36.2 & 68.5 & 40.1 \\
\hline $5 h$ & 77.4 & 52.6 & 61.8 & 52.0 \\
\hline $5 \mathbf{i}$ & 60.8 & 53.4 & 58.6 & 46.9 \\
\hline $5 \mathbf{j}$ & 56.7 & 33.9 & 53.2 & 51.7 \\
\hline $5 \mathbf{k}$ & 60.8 & 39.5 & 56.3 & 42.7 \\
\hline 51 & 78.2 & 47.7 & 69.2 & 48.5 \\
\hline CK & 0.0 & 0.0 & 0.0 & 0.0 \\
\hline
\end{tabular}<smiles>[R]c1ccccc1NC(=O)C(C#N)c1nc(-c2ccccc2)cs1</smiles>

5a, $R_{1}=4-F, R_{2}=H ; \quad 5 b, R_{1}=4-F, R_{2}=3-C_{3} ; \quad 5 c, R_{1}=4-F, R_{2}=4-C_{3} ; 5 d, R_{1}=4-F, R_{2}=2-C l ;$ 5e, $R_{1}=4-F, R_{2}=3-C l ; 5 f, R_{1}=4-F, R_{2}=4-C l ; \quad 5 g, R_{1}=4-F, R_{2}=3-C F_{3} ; 5 \mathbf{h}, R_{1}=4-F, R_{2}=4-C F_{3}$; $5 \mathbf{i}, \mathrm{R}_{1}=2,6-2 \mathrm{~F}, \mathrm{R}_{2}=\mathrm{H} ; 5 \mathbf{j}, \mathrm{R}_{1}=2,6-2 \mathrm{~F}, \mathrm{R}_{2}=2-\mathrm{CH}_{3} ; \mathbf{5 k}, \mathrm{R}_{1}=2,6-\mathrm{F}, \mathrm{R}_{2}=4-\mathrm{Cl} ; 5 \mathrm{I}, \mathrm{R}_{1}=2,6-2 \mathrm{~F}, \mathrm{R}_{2}=4-\mathrm{CF}_{3}$;

Scheme-I: Synthetic route for compounds $\mathbf{5 a - I}$

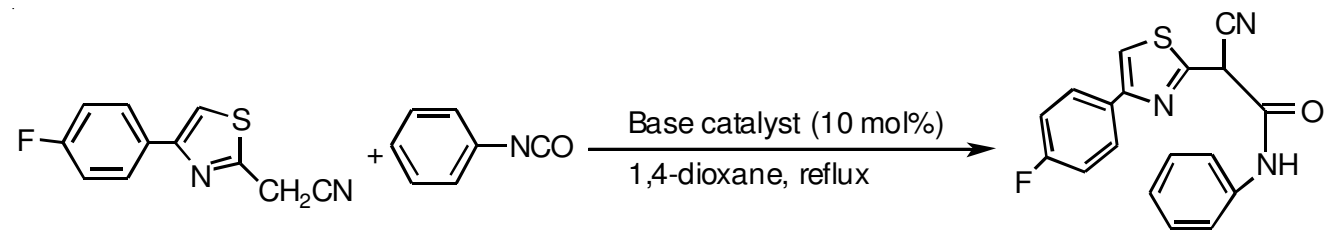

$3 a$

Scheme-II: Reactions of compound 3a with PhNCO catalyzed by organic bases 


\section{Conclusion}

In summary, a series of novel amide derivatives containing thiazole moiety (5) were synthesized via a coupling reaction of [4-(substituted phenyl)thiazol-2-yl] acetonitrile and aryl isocyanates catalyzed by organic bases. The results of preliminary bioassay indicated that these compounds possess certain herbicidal activity against Echinochloa crusgalli and Amaranthus ascedense and could be further developed as potential herbicides.

\section{ACKNOWLEDGEMENTS}

This work was funded by National Natural Science Foundation of China (No. 30900959) and the Key Laboratory of Green Fine Chemistry and Application, Hangzhou, Zhejiang, PR China (No. KFJ2011005).

\section{REFERENCES}

1. X.H. Liu, Y.X. Shi, Y. Ma, C.Y. Zhang, W.L. Dong, P. Li, B.L. Wang, B.J. Li and Z.M. Li, Eur. J. Med. Chem., 44, 2782 (2009).

2. Y. Sawada, T. Yanai and H. Nakagawa, Pest Manage. Sci., 59, 49 (2003).

3. J.Q. Weng, D.L. Shen, C.X. Tan and X.M. Ou, Chin. J. Org. Chem., 26, 1106 (2006).
4. W.L. Dong, Z.X. Liu, X.H. Liu, Z.M. Li and W.G. Zhao, Eur. J. Med. Chem., 45, 1919 (2010).

5. Z.Z. Zhou, Q. Chen and G.F. Yang, Chin. J. Org. Chem., 28, 1385 (2008).

6. N. Muhammad, N. Muhammad, B. Iftikhar and R. Salma, J. Chem. Soc. Pak., 30, 780 (2008).

7. W. Wang, G.P. Zhang, B.A. Song, H. Wang, L.H. Jin, D.Y. Hu and S. Yang, Chin. J. Org. Chem., 27, 279 (2007).

8. Q.M. Wang, H. Li, Y.H. Li and R.Q. Huang, J. Agric. Food Chem., 52, 1918 (2004).

9. P.C. David, V.E. Roy and T.H. Roy, J. Agric. Food Chem., 29, 640 (1981).

10. R.E. Menzer and J.E. Casida, J. Agric. Food Chem., 13, 102 (1965).

11. X.H. Liu, C.X. Tan and J.Q. Weng, Phosphorus Sulfur Silicon Rel. Elem., 186, 552 (2011).

12. S. Vishnoi, V. Agrawal and V.K. Kasana, J. Agric. Food Chem., 57, 3261 (2009).

13. J.Q. Weng, D.L. Shen and C.X. Tan, Chin. J. Org. Chem., 27, 126 (2007).

14. S. Ozaki, Chem. Rev., 72, 457 (1972).

15. J.Q. Weng, H.J. Liu, X.H. Liu, C.X. Tan and D.L. Shen, Chin. J. Pesticide Sci., 12, 463 (2010).

16. P. Yang, C.X. Tan, D.L. Shen, Q. Fu and X.L. Kong, J. Zhejiang Univ. Technol., 35, 19 (2007).

17. F.L. Yang, Z.J. Liu, X.B. Huang and M.W. Ding, J. Heterocycl. Chem., 41, 77 (2004). 